\title{
HBIM Application to Historical Steel Structures: the Case Study of Lapela Bridge
}

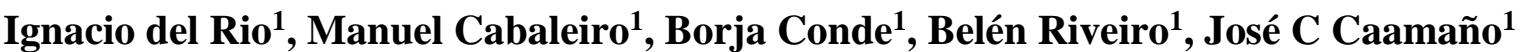 \\ ${ }^{1}$ Department of Materials Engineering, Applied Mechanics and Construction, \\ School of Industrial Engineering, University of Vigo, C.P. 36208, Vigo, Spain. \\ nardric505@gmail.com; mcabaleiro@uvigo.es; bconde@uvigo.es; \\ belenriveiro@uvigo.es; jccaam@uvigo.es
}

\begin{abstract}
Historical steel structures are present all around the world. Besides being a common part of the cultural heritage, many of them are still in service. As an example, we can cite the case of riveted bridges, railway stations, exhibition pavilions, or industrial buildings. The maintenance of these ancient constructions is crucial since they are prone to have suffered significant damage over time due to effects such as corrosion, human actions, or the exposure to heavy loads for which they were not originally conceived. Further, in many cases, these steel structures have to be strengthened in order to adapt them to new uses. HBIM (Historical Buildings Information Modeling) is a new methodology in structural design and construction that could be used as the ideal tool for the maintenance management of these historical structures. HBIM technology is adopted in this paper for structural engineering purposes. Departing from the geometric survey carried out by terrestrial laser scanning, a 3D model is obtained which apart of gathering the main dimensions and details regarding the composition of the structure, it allows collecting all the information concerning the deterioration grade or the different inspections and retrofitting actions performed over time. Thus, by introducing different time stages in the 3D model, the evolution of the structural health over time can be analyzed, which allows the decision-making regarding maintenance and, if required, the undertaking of repairing works. The proposed methodology will be applied to the case study of the Lapela Bridge, in Portugal.
\end{abstract}

Keywords: Maintenance, Laser scanner, Structural health monitoring, 3D Modelling, FEM simulation.

\section{Introduction}

Almost all over the world, we can find historical steel and iron constructions that are still in service or without being in service constitute an important part of the built cultural heritage. There a lot of examples, and some of them even represent an icon for the city or country where they are located, such as the Golden Gate Bridge in San Francisco, the Don Luis I Bridge in Porto, or the Eiffel tower in Paris. The maintenance of these historical steel structures is a very important task since they are prone to suffer damage due to effects such as corrosion, human actions, or the exposure to heavy loads for which they were not originally designed. HBIM (Historical Building Information Modelling) is a new methodology in structural design and construction that can be ideal for the maintenance management of these historical constructions. HBIM [1] consists of the generation and management of all the information of a historical building during its life cycle. It includes the geometrical modelling and numerical simulation and relates geometry with the properties of its components, and even the changes experimented over time. BIM (Building Information Modelling) methodology and HBIM allow creating parametric elements that with the variation of the parameters previously defined encompass all the family of each component. All types of data can be linked to each element, hence creating a database of the building. Moreover, this information can be related to different time phases.

In the case of new buildings, the BIM model is already generated before the building construction itself and all the related documentation is obtained from it. This BIM model is also used for the future maintenance and conservation of the building. However, in the case of historical buildings, they are already in the phase of maintenance or retrofitting when the HBIM model is pretended to be created, so it is always necessary to perform in-situ surveys to get all the needed information, ranging from geometric measurements up to the properties of materials used or its state of conservation. For the geometric data collection stage, and the later generation of 3D models, the laser scanner technique is one of the most suitable tools. 
There are a lot of projects that start with a laser scanning survey to create 3D geometrical models of existent structures, many of them with the final goal of structural analysis [2-9].

In the case of steel structures is worth noting the work of Yang et al [10], which presents a semi-automated approach for extracting geometric information of components of complex steel structures with irregular form from laser scanner data and then creating as-built BIM model based on the information extracted. The work of Basta et al. [11] presents a BIM-based framework for the quantitative evaluation of the deconstruction of steel structures. Laefer \& Truong-Hong [12] proposes a method to automatically identify beam profiles from the point cloud obtained with laser scanning and generating the geometry in a format compatible with BIM. In the specific case of HBIM applied to steel structures, it might be pointed out the work of Donato et al. [13], which presents a framework to obtain an HBIM model of the bridge of Giorgini, in Castiglioni Della Pescaia (Grosseto, Italy). In this work, an integration of many procedures of data collection such as Terrestrial Laser Scanner (TLS), Unmanned Aerial Vehicle (UAV) and through Structure from Motion (SfM) techniques by means of processes derived from the Computer Vision, in order to achieve a full and detailed point cloud as reference for the development of the HBIM model. Also, the work of Morganti et al. [14] where the goal is to define a methodology for the study of steel buildings of the XX century in Italy, considering their specific characteristics and hybrid building nature.

The objective of this work is to propose a methodology for the application of HBIM tools to historical steel and iron structures with the aim of their maintenance management. This HBIM model will collect not only the 3D geometrical model but also all the data necessary to be able to perform maintenance management efficiently, as well as numerical simulation of the mechanical behavior trough the FEM method. The proposed methodology will be applied to the case study of the Lapela Bridge, in Portugal.

\section{Methodology}

The methodology, similarly as it was applied for the case of wooden structures in the work of Mol et al. [20], consists of five main steps that can be repeated in different periods, which are: (i) Data compilation, (ii) Modelling of the elements and structure, (iii) Implementation of the data in the model, (iv) FEM analysis of the structure and

(v) Maintenance management (Fig. 1).

In detail, these steps would be:

i. Data compilation: This would be the phase to be performed directly on the building and it includes the following tasks: laser scanning survey, photographs, detailed visual inspection, measuring of thickness of the beams, measuring of the thickness of the paintings, dynamic identification trough e.g. operational modal analysis, etc.

ii. Modeling of the elements and the whole structure: First, a family has to be created for each element of the structure, for example, a family of $L$ beams, $U$ beams, etc. When creating these families, the cross-section of each beam is defined. Moreover, it is possible to create parameters for the dimensions, so their measures can be changed easily. These families have to be created parametrically. The measurements of the different elements are obtained from the point cloud or directly from in-situ measurements. Then, a structural model is created where every element of the building is inserted. Care must be taken at the moment of joining both models in order to avoid future problems in the exportation.

iii. Data linking to the model: Once the model is created, relevant information at the moment of the scanning is assigned to every element or important entity (e.g. a joint). This information is useful for subsequent FEM simulations, structural health monitoring, as well as for maintenance and conservation tasks. The information can be introduced in different ways such as text, numbers, or links to a database. Examples are photographs, point clouds, discrete measurements, state of corrosion, etc. It is important to reference these data to the date of in-situ surveys, so as to compare them with future studies and evaluate the evolution of the structure in this period.

iv. FEM Simulation: Exportation of the geometric 3D model and the material properties to structural analysis software. Needless to say, the loads that the structure will be subjected to according to the current standards, boundary conditions, and analysis settings have to be defined as well. Finally, the FEM analysis is carried out. As outputs of the analysis, displacement, and stress fields are obtained so that it is possible to analyze the current state of the construction. 
v. Maintenance management: At this point, based on the different data capturing along time and the related structural analysis, it is possible to compare the evolution of the structural health state of each element and the structure. For it, the analysis of the different types of data obtained for each phase time will be carried out.
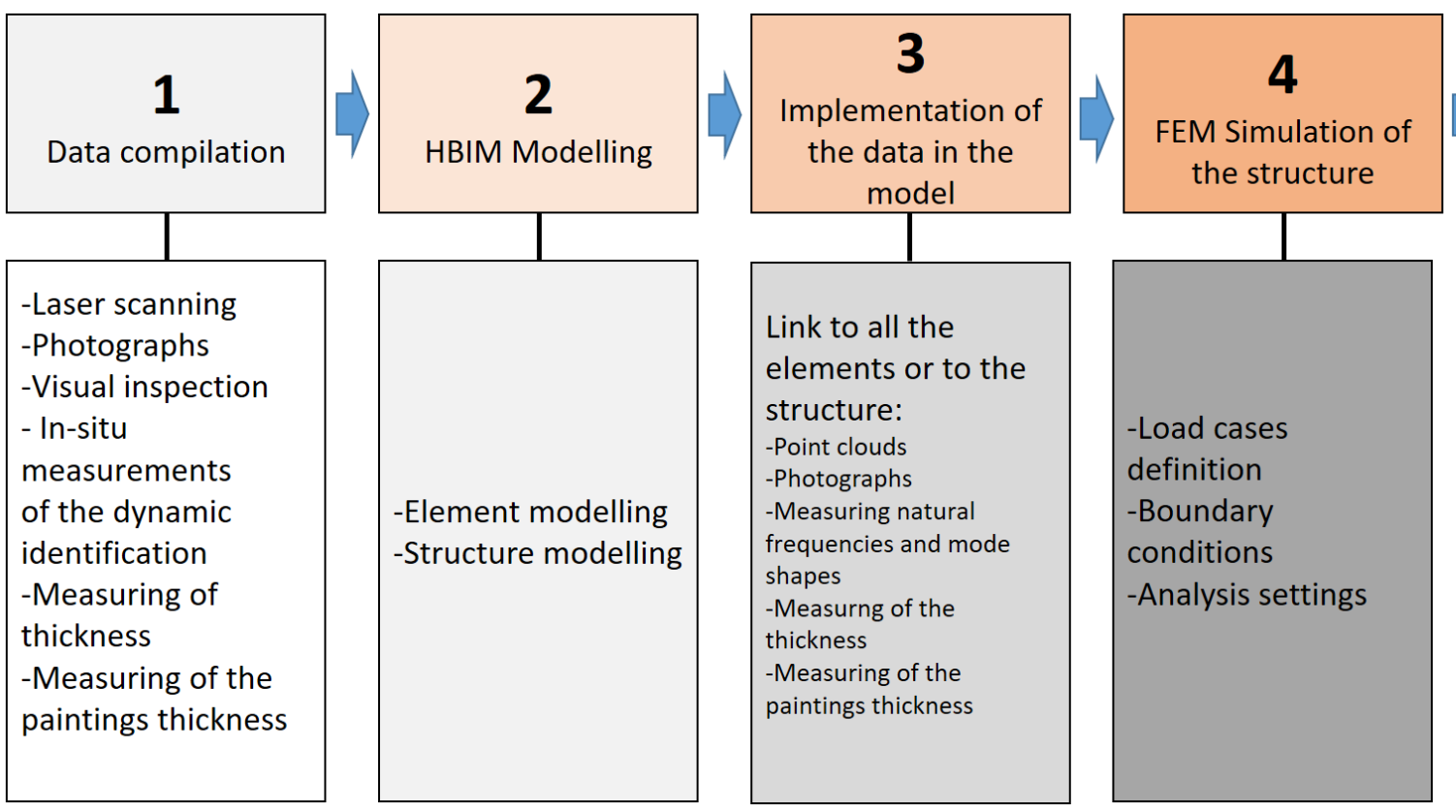

Fig. 1: The workflow of the proposed methodology.

\section{Case study: Lapela Bridge}

The proposed methodology was applied to the Lapela steel bridge in Portugal (Fig. 2). The following equipment was used in the on-site inspection. For the laser scanning, survey, a Faro Focus 3D was employed, which allows measuring distances in a range from 0.6 to 120 meters with an error of $\pm 2 \mathrm{~mm}$. The thickness measurements of the beams were done with a meter Ultrasonic Micrometer model MX-3 (Dakota Ultrasonics) with accuracy \pm 0.01 millimeters, and finally, for the measurement of painting layer thickness a Coating Thickness Meter MEGA-CHECK 20-ST with accuracy $\pm 1 \%$ (100 1000 microns) was used. The point cloud obtained from the terrestrial laser scanning was treated with the software CloudCompare 2.10, while the HBIM modeling stage was performed using Revit 2018. Structural analysis was finally carried out resorting to ANSYS software. 

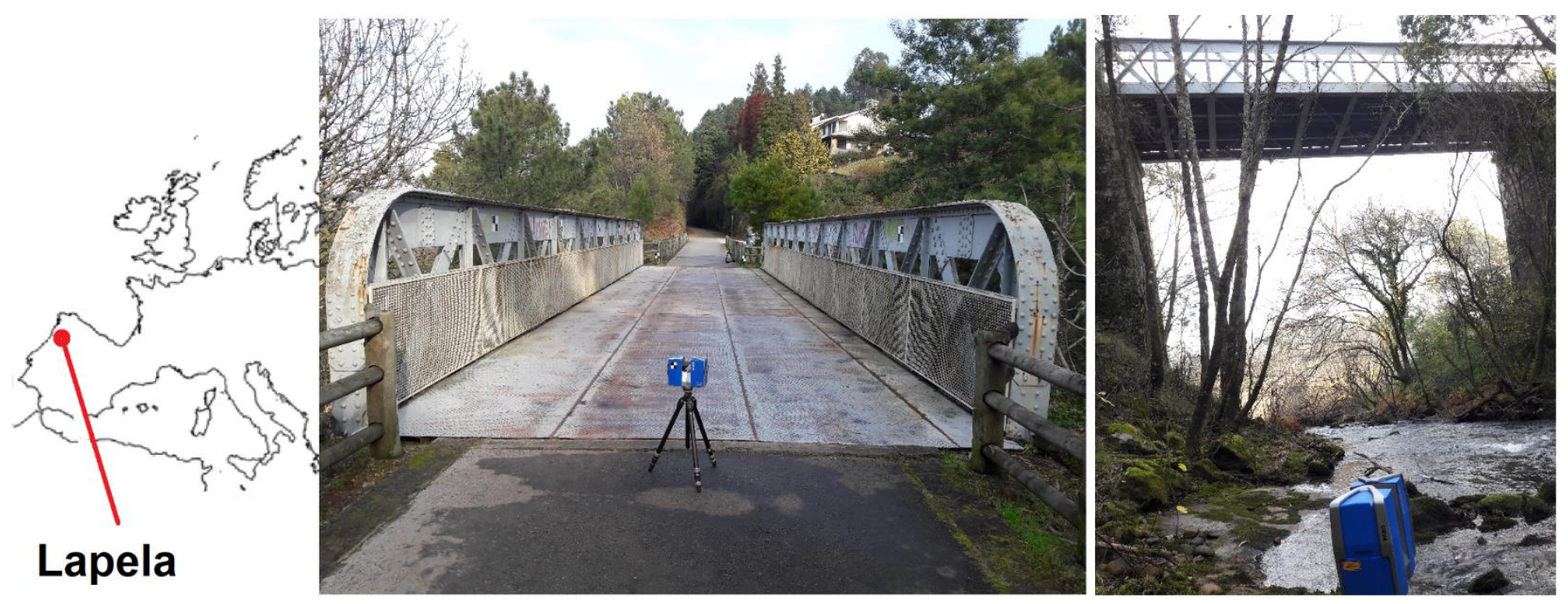

Fig. 2: Location and photographs of Lapela Bridge.

The Lapela Bridge belonged to the old railway line between Valença and Monçao, in Portugal, which was open in 1915 and closed down in 1989. This railway section, which has a length of $22 \mathrm{Km}$, was converted into a greenway in 2004. In 2017 this greenway was awarded as the third-best greenway of Europe, award promoted by the European Greenways Association (E.G.W.A.) and the European Union. The steel bridge, together with the medieval tower are the two architectonic emblems of this greenway and this zone near the Miño river, in the border with Spain. The bridge is usually used only by bicycles and pedestrians, but there is the possibility of being crossed by cars in case of emergency. The structure has a single span with a length of 20 meters and a width of 5 meters. It is formed by different types of steel beams and sheets joined by rivets. 


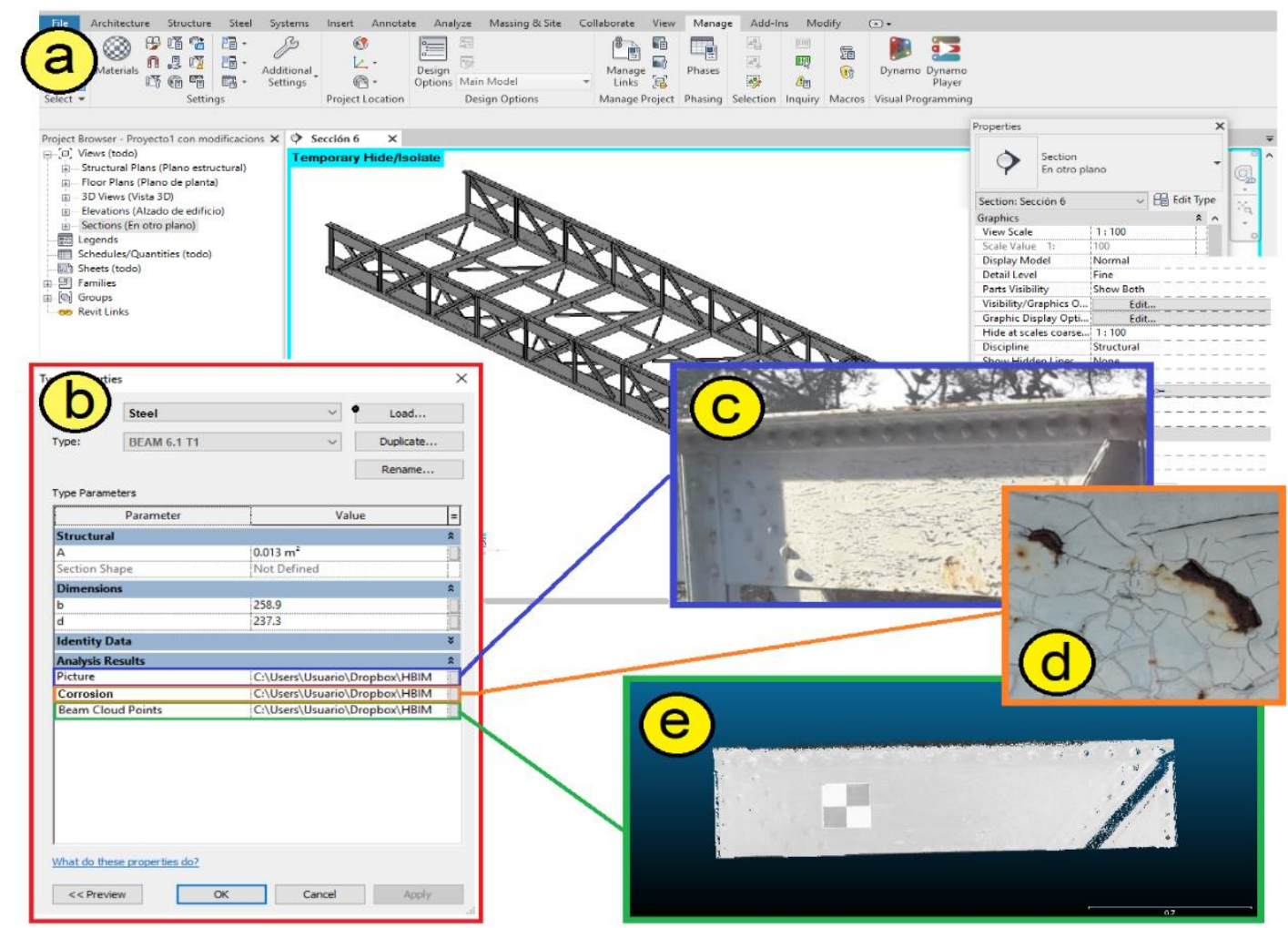

Fig. 3: Example of the data linked to an element in the HBIM model: a) the main screen for the interaction with the information regarding the HBIM modeling of the structure, b) chart of information with links, c) photograph of the beam, d) detail of corrosion, e) beam point cloud.

In order to obtain the geometry of the bridge, a total of 8 scans were carried out, out of which 5 are of the upper part and 3 correspond to the lower part. Each scan has an average of 15,000,000 points with a resolution of $6.0 \mathrm{~mm}$ at a distance of $10 \mathrm{~m}$. From the point clouds, and using the software Cloudcompare to get the relevant measurements, the 3D model was created. Ten families of beam profiles were defined, where each family was used an average of 15 times for the definition of the whole structure.

The corresponding information obtained from the test and the on-site inspections were also linked to each element. This information reads (i) thickness measurement, (ii) photography register of each element, (iii) isolated point cloud of the element, and (iv) analysis of the corrosion state of the element (Fig. 3). Each type of data was associated, depending on the file size, to a document available by a specific URL or directly in the data of the model of the element. All the information mentioned previously, as well as the 3D model, is related to the specific moment of the data acquisition (the year 2020). 

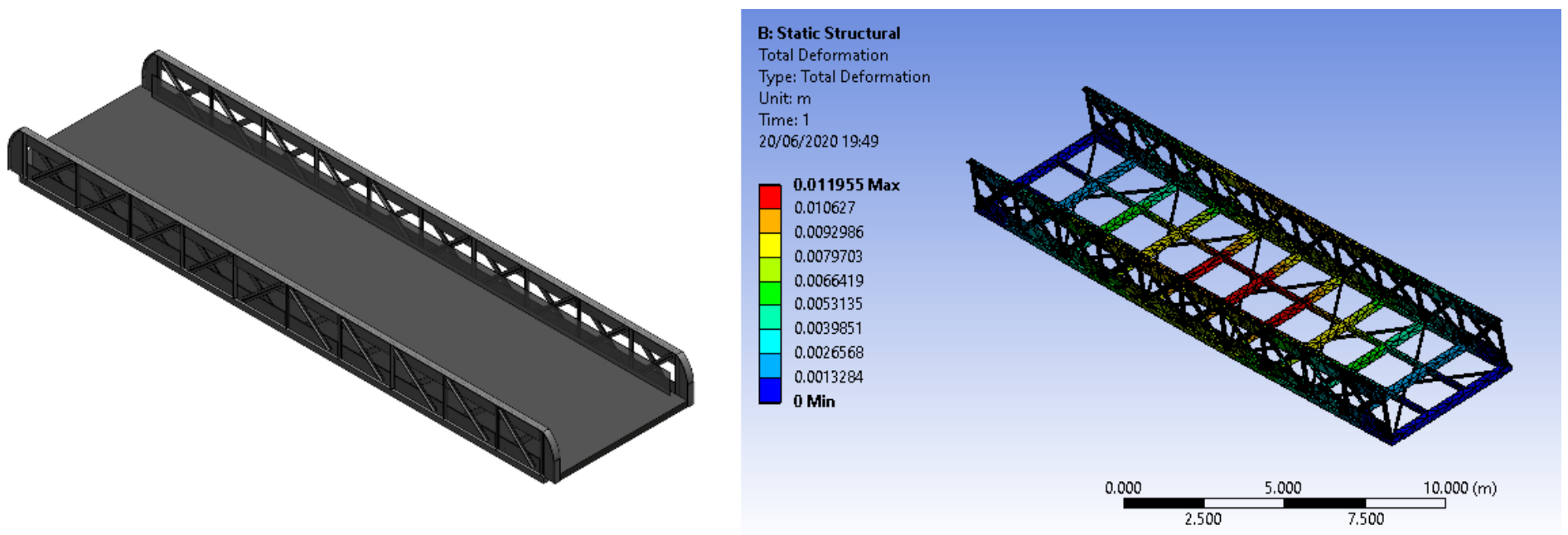

Fig. 4: Geometrical model of the Lapela Bridge and FEM structural analysis.

Subsequently, the 3D model in IFC format was exported to the FEM software ANSYS in order to perform the structural analysis. In this exchange several problems of interchangeability were detected, for example, that ANSYS does not read IFC files, so the *.sat format was used instead. From the obtained results the current condition of the bridge is evaluated. All the information is stored, and available for future studies so as to perform the analysis of the structural health evolution of the bridge.

\section{Conclusion}

HBIM (historical building information modeling) is a new methodology in structural design and construction which has been tested as a tool for the maintenance management of historical steel structures. This tool allows us to perform the geometrical modelling of the construction from the data acquired in-situ with terrestrial laser scanning or other techniques.

HBIM allows relating the geometry of the building with the properties of its componets, also recording the changes experienced over time. Furthermore, from the generated HBIM model, FEM-based structural analysis can be carried out, although the exchange of information between the HBIM models and the software of structural analysis is not perfect yet under the umbrella of the IFC format. Further, with HBIM we can create parametric elements for the family of each element. Having all the information of the structure associated with different periods, this allows us to compare and analyze the evolution of the structural health of the construction, being able to do better maintenance management and taking the most reliable actions of maintenance and intervention.

Future interventions on the Lapela Bridge will allow us to compare the evolution of the structural health of the construction and thus testing the tool of comparison over time based on the HBIM technology. Besides, future works might look for formats and methodologies that allow a reliable exchange of information between HBIM models and FEM software.

\section{Acknowledgements}

This work has been partially supported by the Spanish Ministry of Science, Innovation and Universities through the project Ref. RTI2018-095893-B-C21, and the SIRMA project, which is co-financed by the INTERREG Atlantic Area Programme through the European Regional Development Fund (ERDF) with application code: EAPA_826/2018. 


\section{References}

[1] F.J. López, P.M. Lerones, J. Llamas, J. Gómez-García-Bermejo, E. Zalama, (2018). A review of heritage building information modelling (H-BIM). Multimodal Technologies and Interaction, 2(2), 21.

[2] M. Cabaleiro, B. Riveiro, P. Arias, J.C. Caamano, (2015) Algorithm for beam deformation modeling from LiDAR data, Measurement 76 20-31.

[3] J. Herraez, P. Navarro, J.L. Denia, M.T. Martin, J. Rodriguez, Modeling the thickness of vaults in the church of Santa Maria de Magdalena (Valencia, Spain) with laser scanning techniques, J. Cult. Herit. 15 (6) (2014) 679-686.

[4] M. Cabaleiro, B. Riveiro, P. Arias, J.C. Caamano, J.A. Vilan, (2014) Automatic 3D modelling of metal frame connections from LIDAR data for structural engineering purposes, ISPRS J. Photogramm. Remote Sens. 96 47-56.

[5] X. Yin, H. Liu, Y. Chen, M. Al-Hussein, (2019) Building information modelling for off-site construction: review and future directions, Autom. Constr. 101 72-91.

[6] R. Santos, A.A. Costa, J.D. Silvestre, L. Pyl, (2019) Informetric analysis and review of literature on the role of BIM in sustainable construction, Autom. Constr. 103 221-234.

[7] F. Garzia, D. Costantino, V. Baiocchi, Security and safety management and role of laser scanning in unique and peculiar cultural heritage sites such as the papal basilica and the sacred convent of Saint Francis in Assisi in Italy, International Journal of Heritage Architecture 2 (2) 271-282.

[8] J. Cuartero, M. Cabaleiro, H. S. Sousa, \& J. M. Branco, (2019). Tridimensional parametric model for prediction of structural safety of existing timber roofs using laser scanner and drilling resistance tests. Engineering Structures, 185, 58-67.

[9] L.J. Sanchez-Aparicio, B. Riveiro, D. Gonzalez-Aguilera, L.F. Ramos, The combination of geomatic approaches and operational modal analysis to improve calibration of finite element models: a case of study in Saint Torcato church (Guimaraes, Portugal), Constr. Build. Mater. 70 (2014).

[10] Yang, L., Cheng, J. C., \& Wang, Q. (2020). Semi-automated generation of parametric BIM for steel structures based on terrestrial laser scanning data. Automation in Construction, 112, 103037.

[11] A. Basta, M. H. Serror, \& M. Marzouk, (2020). A BIM-based framework for quantitative assessment of steel structure deconstructability. Automation in Construction, 111, 103064.

[12] D. F. Laefer, \& L. Truong-Hong (2017). Toward automatic generation of 3D steel structures for building information modelling. Automation in Construction, 74, 66-77.

[13] V. Donato, C. G. Biagini, Bertini, \& F. Marsugli, Challenges and opportunities for the implementation of h-bim with regards to historical infrastructures: a case study of the ponte giorgini in castiglione della pescaia (grossetoitaly). International Archives of the Photogrammetry, Remote Sensing \& Spatial Information Sciences, (2017). 42.

[14]R. Morganti, A. Tosone, D. Di Donato, \& M. Abita, (2019). Hbim and the 20th century steel building heritage-a procedure suitable for the construction history in italy. International Archives of the Photogrammetry, Remote Sensing \& Spatial Information Sciences.

[15]A. Mol, M. Cabaleiro, H. S. Sousa, \& J. M. Branco. (2020). HBIM for storing life-cycle data regarding decay and damage in existing timber structures. Automation in Construction, 117, 103262. 